\title{
El látex. Un importante aeroalergeno implicado en el asma ocupacional
}

\section{Latex. An important aeroallergen involved in occupational asthma}

\section{S. Quirce' ${ }^{1}$ J.M. Olaguíbel' ${ }^{2}$ M.J. Álvarez², A.I. Tabar ${ }^{2}$}

\section{RESUMEN}

El látex ha pasado a ser uno de los agentes etiológicos más frecuentes de asma ocupacional en la última década, especialmente entre el personal sanitario. La prevalencia de asma ocupacional por látex en las distintas profesiones afectadas varía entre el 2,5 y el $10 \%$, cifras que dan una idea de la gravedad del problema. Las cifras que dan una idea de la gravedad del problema. Las proteínas del látex, adsorbidas en el polvo de almidón de
maíz, utilizado como lubricante, son las responsables del maíz, utilizado como lubricante, son las responsables del asma por guantes de látex. Este polvo de almidón de maíz que recubre el interior de los guantes actuaría como un vehículo para la dispersión de las proteínas distintos tamaños de partículas. Los niveles de aeroalerdistintos tánaños de particus genos de látex en ensiblización y de desencadenar sintomas no están bien definidos. La determinación de estos niveles de exposición al látex es complicada, ya que el látex se trata de una mezcla compleja de alergenos con diferente estabilidad y biodisponibilidad. El bioaerosol de látex se produce principalmente por el uso activo de guantes empolvados, pero las moquetas o alfombras y las tapicerías actúan como importantes reservorios del alergeno. Además, el desplaimpor tantes reservorios del alergen. Además, el desplazamiento de tración ambiental de látex constituye una importante vía de dispersión del alergeno, así como probablemente lo sistemas de ventilación. Como en otros casos de asma ocupacional el diagnóstico en muchas ocasiones requiere la realización de una prueba de provocación bronquial. Las pruebas de provocación específicas por vía inhalatoria con látex se han realizado por diversos métodos, que pueden agruparse en aquellos que utilizan un extracto acuoso de látex y los consistentes en manipular extracto accooso de látex y los consistentes en manipula o sacudir guantes, que por tanto, generan un aerosol de polvo. Cada método tiene sus ventajas e inconvenientes según se describe. Una vez que el paciente sensibilizado inicia las manifestaciones de la enfermedad, tiene un mal pronóstico si no se aplican medidas encaminadas a reducir al máximo la exposición al látex.

An. sis. sanit. Navar. 2003; 26 (Supl. 2): 81-95

\section{ABSTRACT}

Latex has become one of the most frequent aetiological agents of occupational asthma in the last decade, especially amongst health personnel. The prevalence of occupational asthma due to latex in the prevalence of occupational asthma due to latex in the $10 \%$, figures that give an idea of the magnitude of the $10 \%$, figures that give an idea of the magnitude of the
problem. Latex proteins, absorbed in the dust of cornstarch, used as a lubricant, are responsible for asthma due to latex gloves. This cornstarch dust that covers the inside of the gloves acts as a vehicle for dispersing allergenic proteins. Latex aeroallergens are found in particles of different dimensions. The levels of found in particles of prifere levels of latex aeroallergens in the environment capable of causing sensitization and of unleashing symptoms are not well defined. The determination of these levels o exposure to latex is complicated, since with latex one is dealing with a complex mixture of allergens with differing stability and bio-availability. The bioaerosol of latex is mainly produced by the active use of powdered gloves, but rugs or carpets and coverings act as important reservoirs of the allergen. Besides, the movement of people from areas with a high environmental concentration of latex constitutes an important means of dispersing the allergen, and important means of dispersing the allergen, and
probably ventilation systems as well. As in other cases probably ventilation systems as well. As in other cases of occupational asthma, diagnosis in many case
requires that a bronchial challenge be realized. The specific challenge tests through inhalation with latex have been carried out by different methods, which can be grouped by those that use an aqueous extract of latex and those that consist in handling or shaking gloves, which thus generate a dust aerosol. Each gloves, which thus generate a dust aerosol. Each method has its advantages and drawbacks as described. Once the sensitized patient begins
manifestations of the disease, his prognosis is poor if measures aimed at a maximum reduction of exposure to latex are not applied. 1. Sección de Alergología. Fundación Jiménez
Díaz. Madrid

2. .Sección de Alergología. Hospital Virgen del Camino. Pamplona

\author{
Correspondencia: \\ D. Santiago Quirce \\ Unidad de Alergología \\ Fundación Jiménez Díaz \\ Avda. Reyes Católicos, 2 \\ 28040 Madrid
}




\section{INTRODUCCIÓN}

En 1986 Carrillo y col $^{1}$ describieron a una médico atópica de 35 años que presentaba, además de urticaria de contacto por sensibilización al látex, rinitis y disnea al abrir las bolsas de los guantes de látex, demostrando la implicación de un mecanismo alérgico mediado por IgE frente a proteínas del látex. De ser completamente desconocido como causa de asma hace sólo 15 años, el látex ha pasado a ser uno de los agentes etiológicos más frecuentes de asma ocupacional en la última década, especialmente entre el personal sanitario $^{2}$. El programa de vigilancia epidemiológica de asma ocupacional en el Reino Unido (SWORD) ha detectado a látex como una de las causas más frecuente de asma ocupacional ${ }^{3}$ y en Sudáfrica ha sido identificado como la causa más frecuente de asma profesional entre 1996 y $1998^{4}$.

Aunque inicialmente se intentó relacionar el asma inducida por guantes de látex que padecía un técnico de laboratorio con un terpeno bicíclico que desprendían los guantes ${ }^{5}$, pronto se pudo comprobar que eran las proteínas del látex, adsorbidas en el polvo de almidón de maíz utilizado como lubricante, las responsables del asma por guantes de látex ${ }^{6,7}$. Este polvo de almidón de maíz que recubre el interior de los guantes actuaría como un vehículo para la dispersión de las proteínas alergénicas del látex. En la actualidad, el látex es reconocido como un potente aeroalergeno que causa una importante morbilidad, sobre todo entre los sujetos profesionalmente expuestos.

Baur y Jäger ${ }^{6}$ estudiaron a 10 trabajadores sanitarios (9 cirujanos o enfermeras de quirófano y un neurólogo) y a un paciente que presentaban síntomas respiratorios con látex. Para confirmar la relevancia de la exposición al polvo de los guantes en los síntomas respiratorios de estos pacientes, realizaron pruebas de exposición con los guantes de látex y de otro material alternativo. Encontraron que la manipulación de uno a 20 pares de guantes de látex durante 4 a 30 minutos producía rinitis en todos los casos, y en 4 pacientes indujo crisis de asma. Estos datos con- firmaban que los alergenos del látex no sólo causaban síntomas por contacto cutáneo, sino que también podían actuar como aeroalergenos tras ser transferidos al polvo de los guantes, que posteriormente sería aerosolizado al manipular los guantes. Hoy en día se considera que el látex es un aeroalergeno muy importante en el medio hospitalario y que la principal fuente de exposición al látex en el personal sanitario la constituyen los guantes de látex con polvo.

Marcos $\mathrm{y} \mathrm{col}^{8}$ describieron una enfermera de quirófano, no atópica, que presentaba urticaria de contacto, rinitis, conjuntivitis y asma con los guantes de látex, y documentaron de forma concluyente mediante pruebas cutáneas en prick, detección de IgE específica, mediciones seriadas del pico de flujo espiratorio (PEF) y provocación bronquial específica con extracto de guantes, la implicación del látex en los síntomas alérgicos de esta paciente. Posteriormente, Jaeger y $\mathrm{col}^{9}$ incidieron sobre la presencia de síntomas respiratorios en los trabajadores sanitarios alérgicos al látex. Estos autores estudiaron a 70 pacientes (64 eran trabajadores sanitarios) que presentaban reacciones alérgicas mediadas por IgE causadas por artículos de látex. Todos los pacientes presentaban urticaria, 36 rinitis, 31 conjuntivitis, 22 disnea y 7 reacciones sistémicas, y 4 pacientes tuvieron reacciones graves durante la cirugía. Cinco de los 18 pacientes en los que se realizó provocación bronquial específica con guantes de látex empolvados mostraron un aumento significativo de la resistencia de la vía aérea, 16 rinitis y 2 presentaron reacciones sistémicas, confirmando el potencial asmogénico del aerosol de proteínas del látex unidas al almidón de maíz. En la Clínica Mayo en EE.UU. se describieron 49 empleados de este centro médico que presentaban alergia respiratoria al látex (rinitis y asma) cuando trabajaban en áreas donde los guantes de látex se utilizaban profusamente ${ }^{10}$. A la luz de estos datos, se fue consolidando el concepto de que el asma es una manifestación clínica muy frecuente en los trabajadores sanitarios alérgicos al látex. 


\section{PREVALENCIA}

Tarlo y col $^{11}$ describieron a una paciente de 33 años que trabajaba inflando guantes de látex recubiertos con polvo en una empresa de suministros médicos y que desarrolló síntomas de asma en su trabajo. Está paciente fue diagnosticada de asma ocupacional por látex basándose en las pruebas cutáneas positivas, en los registros del PEF y en los cambios de la hiperreactividad bronquial a metacolina relacionados con la exposición laboral. El estudio se amplió a los 81 trabajadores de esta empresa, encontrándose pruebas cutáneas positivas al látex en el $11 \%$ de los trabajadores evaluados (7/64), y el $6 \%$ de los empleados estudiados (3/50) presentaban hallazgos compatibles con asma ocupacional en las pruebas de función pulmonar realizadas (cambios en $\mathrm{PEF}$, espirometría o PC20 metacolina). Vandenplas y col $^{12}$ estudiaron los empleados de un hospital general incluyendo 201 enfermeras, 50 trabajadores en la limpieza y 38 técnicos de laboratorio. En la fase inicial de este estudio, realizaron un cuestionario y pruebas cutáneas en prick con látex y aeroalergenos comunes a 273 de los 289 (94\%) trabajadores de la plantilla seleccionada. Trece de los 273 trabajadores (4,7\%) tenían pruebas cutáneas positivas al látex. Todos los pacientes sensibilizados al látex presentaban urticaria de contacto con los guantes de látex, asociándose con rinoconjuntivitis en 12 pacientes y con asma en 5 . Entre los trabajadores con pruebas cutáneas negativas al látex ninguno tenía historia sugestiva de asma ocupacional. A todos ellos se les realizó test de provocación bronquial específica con guantes de látex en el laboratorio y 7 de ellos presentaron una respuesta asmática (inmediata en 4 casos y dual en 3 casos). Por tanto, la prevalencia de asma ocupacional por látex, confirmada por pruebas de provocación, fue del 2,5\% de los empleados hospitalarios.

El asma ocupacional por látex también se ha descrito en trabajadores de una fábrica de muñecas de látex ${ }^{13}$. Pisati y col ${ }^{14}$ realizaron un estudio en 10 trabajadores de una fábrica textil donde se utilizaban y trenzaban fibras de látex (empolvadas con talco para reducir la pegajosidad del látex natural), nylon y poliamida para realizar elásticos para la ropa interior. Dos de los trabajadores tenían síntomas cutáneos, oculares y respiratorios (nasales o bronquiales) relacionados con el trabajo. Se realizaron provocaciones bronquiales específicas mediante nebulización de extractos de polvo de talco con látex en estos dos empleados, mostrando ambos estornudos, rinorrea, y flash facial. Uno de los trabajadores también presentó una disminución del VEMS del $32 \%$ a los 10 minutos de la provocación. La exposición a un extracto de polvo de talco puro no indujo ninguna respuesta. Estos datos indican que el polvo de talco también puede absorber los alergenos de látex y producir síntomas por vía inhalatoria. También se han descrito casos aislados de asma ocupacional por látex en una costurera ${ }^{15} \mathrm{y}$ en un empleado en una fábrica de chicles $^{16}$. Por tanto, la prevalencia de asma ocupacional por látex en las distintas profesiones afectadas varía entre el 2,5 y el $10 \%{ }^{12-14}$, cifras que dan una idea de la gravedad del problema.

\section{DISPERSIÓN AMBIENTAL DEL} LÁTEX

Se ha sugerido que la exposición por vía inhalatoria al látex podría ser la que con más facilidad indujera la producción de IgE, actuando como la vía principal de sensibilización en áreas en las que existen altas concentraciones de partículas de látex aerosolizadas en el ambiente, como ciertas zonas hospitalarias ${ }^{17}$. No obstante, aunque el asma por látex generalmente se produce por inhalación de partículas aerosolizadas de látex ${ }^{18}$, en ciertos casos el asma puede formar parte de una reacción sistémica inducida por este alergeno ${ }^{19}$.

Como hemos visto, la posibilidad de que las proteínas de látex pudieran actuar como aeroalergenos, una vez absorbidas a ciertas partículas transportadoras como el polvo de almidón de maíz del interior de los guantes o el talco, había sido sugerida por varios autores. Se suponía que los alergenos del látex pasarían de los guantes al polvo que recubre su interior, viéndose aerosolizados al manipular y utilizar los guantes. Por tanto, en el medio hospitalario y en los centros sanitarios el látex se 
comportaría como un aeroalergeno muy importante.

Tomaciz y $\operatorname{col}^{20}$ demostraron que las proteínas del látex se fijan al polvo de almidón de maíz, lo que apoya la relación causal entre los síntomas que experimentan los pacientes alérgicos al látex que se ven expuestos por vía inhalatoria a partículas aerosolizadas de los objetos de látex empolvados. Para ello, estudiaron la capacidad del polvo de almidón de maíz para absorber las proteínas del látex y evaluaron la actividad alergénica de las proteínas unidas a este polvo. La alergenicidad se investigó mediante ensayos de inhibición competitiva de la unión de IgE específica de pacientes alérgicos al látex al propio látex fijado en la fase sólida. Se comparó la actividad alergénica del polvo de los guantes, de polvo de almidón de maíz incubado con extracto de látex y de un polvo de maíz puro no contaminado con látex. Las dos muestras de polvo de maíz expuestos al látex inhibieron de forma dosis-dependiente la fijación específica de los anticuerpos IgE a las proteínas del látex. Por el contrario, el almidón de maíz no contaminado con látex no producía ninguna inhibición. El análisis del almidón de maíz obtenido de los guantes de látex mostraba que la cantidad de proteína de látex que se fija al almidón depende tanto de la cantidad total de proteína presente en el objeto de látex como de la cantidad de almidón que se añade a dicho producto. Estos autores sugieren que es posible que las múltiples proteínas alergénicas que quedan firmemente retenidas en las partículas del almidón de maíz serían más eficaces en causar un entrecruzamiento efectivo de las moléculas de IgE unidas a los mastocitos que los propios alergenos del látex solubilizados. Esto explicaría por qué los síntomas alérgicos respiratorios podrían ser desencadenados en pacientes sensibilizados por la exposición incluso a cantidades mínimas de polvo de almidón dispersado al ambiente, por ejemplo al abrir una bolsa de guantes.

Los niveles de aeroalergenos de látex en el ambiente capaces de causar sensibilización y de desencadenar síntomas no están bien definidos. La determinación de estos niveles de exposición al látex es complicada, ya que el látex se trata de una mezcla compleja de alergenos con diferente estabilidad y biodisponibilidad. Los estudios de cuantificación o medición de la concentración ambiental de aeroalergenos del látex se han realizado en ambientes sanitarios y hospitalarios, clínicas dentales y en las plantas industriales de procesamiento del látex ${ }^{21-29}$. Swanson y $\mathrm{col}^{21}$ estudiaron la concentración, variabilidad, así como el diámetro aerodinámico medio de los aeroalergenos del látex en un gran centro médico, como es la Clínica Mayo (EE.UU.). Para ello, tomaron muestras ambientales en distintas áreas hospitalarias utilizando medidores ambientales de área (Air Sentinel) y bombas personales. La concentración de aeroalergeno de látex retenida en los filtros se determinó mediante ensayos de inhibición de ELISA IgE utilizando el suero de pacientes alérgicos al látex. La concentración de aeroalergeno de látex en 11 áreas hospitalarias donde se utilizaban guantes de látex empolvados con mucha frecuencia varió entre 13 y $208 \mathrm{ng} / \mathrm{m}^{3}$, y en aquellas áreas donde apenas se utilizaban los guantes de látex las concentraciones variaban entre 0,3 y $1,8 \mathrm{ng} / \mathrm{m}^{3}$. La instalación y utilización de una estación de flujo laminar para cambiarse los guantes no redujo los niveles de aeroalergeno de látex. Además, se detectaban grandes cantidades de alergenos de látex en las batas utilizadas en los laboratorios y en las zonas de lavado pre-anestésicas, así como en los propios laboratorios. Las concentraciones de aeroalergeno de látex medidas con bombas de muestreo personales en zonas de gran utilización de guantes oscilaron entre 8 y $974 \mathrm{ng} / \mathrm{m}^{3}$. La mayor exposición se producía probablemente al calzarse o cambiarse los guantes debido a la resuspensión de los reservorios de polvo presentes en las salas y en la ropa. Los aeroalergenos de látex se encontraban en distintos tamaños de partículas, tal como se determinó mediante un impactador de cascada, pero la mayor parte (80\%) se detectaban en partículas mayores de $7 \mu \mathrm{m}$ de diámetro medio de masa aerodinámica. Los resultados de la inmunodetección con muestras ambientales de los filtros, mostraban que los aeroalergenos del látex son principalmente proteínas de 
alto peso molecular. Baur y $\mathrm{col}^{25}$ encuentran que niveles de aeroalergeno de látex a partir de $0,6 \mathrm{ng} / \mathrm{m}^{3}$ en el aire ambiental de salas de hospital se asocian con un mayor número de trabajadores sensibilizados y con el desarrollo de síntomas en los pacientes sensibilizados. Recientemente, Charous y $\mathrm{col}^{28}$ han confirmado que el bioaerosol de látex se produce principalmente por el uso activo de guantes empolvados, pero las moquetas o alfombras y las tapicerías actúan como importantes reservorios del alergeno. Además, estos autores describen que el desplazamiento de las personas desde zonas con gran concentración ambiental de látex constituye una importante vía de dispersión del alergeno, así como probablemente los sistemas de ventilación.

Por otra parte, en dos estudios se ha apuntado la presencia de alergenos de látex en muestras de aire urbano ${ }^{30,31}$, probablemente provenientes de partículas del desgaste de neumáticos, sugiriendo que podrían influir en el aumento de la prevalencia de la sensibilización y del asma por látex. No obstante, en estos estudios no se ha tenido en cuenta la posible reactividad cruzada entre alergenos del látex y pólenes.

\section{DIAGNÓSTICO DEL ASMA POR LÁTEX}

La prueba más fiable para confirmar el diagnóstico de asma ocupacional por látex, al igual que ocurre con otros alergenos laborales, es la provocación bronquial específica. Sin embargo, dado que esta prueba es compleja y requiere equipamiento adecuado y personal entrenado en su realización, sólo se lleva a cabo en algunos centros. Por ello, el diagnóstico de asma ocupacional con alergenos de alto peso molecular, como es el caso del látex, con frecuencia se realiza basándose en una historia clínica compatible, la demostración de hiperreactividad bronquial inespecífica y de pruebas cutáneas de hipersensibilidad inmediata positivas. Sin embargo, la rentabilidad diagnóstica de esta estrategia no está bien establecida.

Vandenplas y $\mathrm{col}^{32}$ han estudiado recientemente la validez de la historia clí- nica, las pruebas cutáneas y la medición de la hiperreactividad bronquial a histamina en el diagnóstico del asma ocupacional causada por látex, comparándolo con la provocación bronquial específica con látex. Para ello evaluaron a 45 pacientes remitidos consecutivamente a su clínica para el estudio de posible asma ocupacional causada por látex. Estos pacientes fueron sometidos a un detallado interrogatorio clínico y laboral, pruebas cutáneas en prick frente a látex, prueba de inhalación bronquial con histamina y test de provocación inhalatoria con guantes de látex empolvados. La historia clínica obtenida fue valorada de forma retrospectiva por 4 médicos especialistas que desconocían el resultado de las pruebas realizadas y que dieron su opinión sobre la probabilidad de que el paciente tuviera asma ocupacional por látex. La historia clínica, las pruebas cutáneas en prick con látex y la medición de la hiperreactividad bronquial a histamina mostraron una alta sensibilidad $(87 \%$, $100 \%$ y $90 \%$, respectivamente) pero una baja especificidad (14\%, $21 \%$ y $7 \%$ ) al compararlas con los resultados de la provocación bronquial específica. El análisis mediante regresión logística mostró que la combinación de los resultados de las pruebas cutáneas con látex y la historia clínica aumentaban el valor predictivo negativo del 50 al $71 \%$, mientras que el valor predictivo positivo permanecía básicamente igual (75 y 76\%). Como conclusión de este estudio los autores señalaban que la provocación bronquial específica es necesaria para confirmar el diagnóstico de asma causada por látex. Baur $\mathrm{y} \mathrm{col}^{33}$ realizaron un estudio en un grupo de trabajadores sanitarios con sospecha de asma ocupacional por látex e investigaron la fiabilidad de un cuestionario estandarizado combinado con la entrevista por un médico especialista. Encontraron que la historia clínica tenía una sensibilidad del $92 \%$, una especificidad del $32 \%$, un valor predictivo positivo del $24 \%$ y un valor predictivo negativo del $94 \%$, comparado con la provocación bronquial específica con guantes de látex.

Existen varios factores que pueden afectar la interpretación de la historia en el caso del asma por látex, como la variabilidad en la intensidad de la exposición, las 
fuentes de exposición indirecta, la presencia de otros alergenos o irritantes en el medio sanitario, etc. De hecho, en el estudio de Vandenplas y col $^{32}$ sólo el $39 \%$ de los pacientes con asma ocupacional por látex identificaron a los guantes de látex como los causantes de su asma.

La presencia de hiperreactividad bronquial a agentes farmacológicos determinada el día previo a la provocación con látex no predice de forma inequívoca el resultado de la provocación con látex. Los resultados del test de histamina tenían un valor predictivo positivo del $68 \%$ y un valor predictivo negativo del $25 \%{ }^{32}$. Sin embargo, la medición de la hiperreactividad bronquial tras la provocación con látex aumenta el valor predictivo negativo hasta el $100 \%$. Por tanto, las pruebas de provocación bronquial con látex, al igual que con otros alergenos ocupacionales, deberían incluir una nueva determinación de la hiperreactividad bronquial a agentes farmacológicos a las 12-24 horas post-provocación, ya que un aumento de la hiperreactividad bronquial es un marcador precoz y sensible de una repuesta positiva en la provocación bronquial específica.

Las pruebas cutáneas con látex tienen una sensibilidad y un valor predictivo negativo del $100 \%$ en el diagnóstico del asma por látex pero tienen una baja especificidad $(21 \%)^{32}$. Por tanto, las pruebas cutáneas son especialmente útiles para excluir el diagnóstico de asma por látex. Por el contrario, las pruebas cutáneas tienen menos utilidad en confirmar el diagnóstico (valor predictivo positivo del $74 \%)$.

El principal inconveniente de la provocación bronquial específica podría ser la pérdida de reactividad tras un largo periodo sin exposición al alergeno, induciendo a resultados falsos negativos. Lemière $\mathrm{y} \mathrm{col}^{34}$ investigaron este tema con varios agentes ocupacionales 2 años después de haber cesado la exposición. Encontraron que el $60 \%$ de los pacientes requerían un aumento de dos veces en la dosis total inhalada de alergeno para inducir la respuesta asmática y el $13 \%$ reaccionaron con la misma dosis. Un $20 \%$ de los pacientes no presentaron reacción asmática pero sí un aumento de la hiperreactividad bronquial inespecífica post-provocación y sólo el 7\% de los pacientes habían perdido por completo la reactividad bronquial al alergeno ${ }^{34}$.

\section{METODOLOGÍA DE LA \\ PROVOCACIÓN BRONQUIAL}

Las pruebas de provocación específicas por vía inhalatoria con látex se han realizado por diversos métodos, que pueden agruparse en aquellos que utilizan un extracto acuoso de látex y los consistentes en manipular o sacudir guantes, que por tanto, generan un aerosol de polvo. Mediante estas técnicas se pueden inducir respuestas de rinoconjuntivitis y broncoespasmo en las personas sensibilizadas, aunque cada método tiene sus ventajas $e$ inconvenientes. La provocación conjunti$\mathrm{val}^{35}$ y la provocación nasal ${ }^{36}$ también se han utilizado en el diagnóstico de alergia a látex, pero por lo general tienen escaso valor en el diagnóstico de patología respiratoria alérgica ocupacional.

La provocación bronquial específica es necesaria en ciertos casos para confirmar el diagnóstico de asma por látex, aunque existe el riesgo de que aparezcan reacciones de broncoespasmo grave o anafilaxia durante la misma ${ }^{37}$. Este riesgo puede deberse en parte a la dificultad en realizar un incremento progresivo de la dosis de alergeno y de comenzar con concentraciones bajas, por lo que debe realizarse siempre en condiciones controladas y por personal experto.

\section{Provocación con extractos acuosos de guante de látex o látex natural}

\section{Con nebulizador}

Esta técnica se realiza mediante la inhalación de un extracto acuoso de guante de látex natural ${ }^{8} \mathrm{o}$ un extracto comercial de látex ${ }^{38}$, realizando previamente una titulación cutánea a punto final para seleccionar la dosis inicial a inhalar.

Marcos y $\mathrm{col}^{8}$ prepararon un extracto del guante de látex, para lo cual se lava el interior del guante empolvado con $10 \mathrm{ml}$ de PBS durante $10 \mathrm{~min}$. El líquido que se obtiene se centrifuga a $1000 \mathrm{~g}$ durante 10 
minutos y finalmente se esteriliza pasándolo a través de un filtro de $0,22 \mathrm{~mm}$. Se realizan diluciones progresivas (generalmente a 1/2) para la titulación cutánea a punto final y la provocación bronquial específica. Se comienza la provocación bronquial con la pápula que produce una respuesta cutánea de habón de $3 \mathrm{~mm}$. Ho y $\mathrm{col}^{38}$ utilizaron este mismo método pero con un extracto comercial de látex en 4 trabajadores sanitarios con sospecha de asma por látex, confirmándose el diagnóstico en 3 de ellos. El equipamiento necesario para realizar este tipo de provocación es más sencillo y se encuentra al alcance de la mayoría de los servicios de alergia.

Siguiendo este método, en la Sección de Alergología del Hospital Virgen del Camino hemos realizado un estudio de provocación bronquial convencional con extracto acuoso de látex en un grupo de pacientes con sospecha de asma ocupacional, que se describe a continuación ${ }^{39}$.

\section{Características de los pacientes}

Se realizaron provocaciones bronquiales específicas en un total de 12 pacientes con sospecha de asma ocupacional por látex, nueve ellos trabajadores sanitarios, dos empleados en industrias relacionadas con el látex y un empleado en limpieza. En todos los pacientes se realizó una historia clínica detallada, incluyendo historia laboral y presencia de otras manifestaciones alérgicas, en especial alergia a frutas. La intensidad de la clínica se valoró de acuerdo con una puntuación combinada de síntomas y de consumo de fármacos (2-10 puntos). Se realizaron pruebas cutáneas con una batería estándar de aeroalergenos habituales, látex, papaína, castaña, kiwi y plátano. También se cuantificaron los niveles de IgE total y específica sérica frente a látex natural (CAP Pharmacia), se realizó provocación conjuntival con látex $(0,1-1$ $\mathrm{mg} / \mathrm{ml}$ ), estudio funcional respiratorio basal y provocación bronquial con metacolina (método abreviado). La provocación bronquial específica se realizó utilizando un nebulizador DeVilbiss 646 con un débito de $0,28 \mathrm{ml} /$ minuto. El extracto utilizado fue un extracto comercial de látex natural con bajo contenido en amoníaco (Bial-Arístegui) y con una concentración total de proteínas de $10 \mathrm{mg} / \mathrm{ml}$. Se administraron concentraciones progresivamente crecientes al doble entre 0,0150 y 2,5 $\mathrm{mg} / \mathrm{ml}$, inhalando el paciente a volumen corriente durante 2 minutos. La concentración inicial se determinó en cada paciente por titulación a punto final $(3 \mathrm{~mm})$ en prick. En dos pacientes se realizó una inducción de esputo con salino hipertónico en situación basal y a las 24 horas de la provocación con látex, con recuento celular diferencial. En uno de ellos se repitió la provocación bronquial e inducción de esputo tras un año de permanecer de baja laboral sin contacto con látex. Los pacientes fueron posteriormente tratados de acuerdo a criterios clínicos habituales, y revisados periódicamente determinando su situación clínica.

Todos los pacientes, además de síntomas de asma, presentaban urticaria de contacto y rinoconjuntivitis con la exposición al látex y 3 habían presentado episodios de anafilaxia. La primera manifestación en aparecer fue la rinitis y urticaria de contacto de forma simultánea en 6 pacientes, la urticaria de contacto en 2 pacientes, y en 3 pacientes debutaron juntos la rinitis, la urticaria de contacto y el asma bronquial. La forma de debut en un paciente fue un episodio de anafilaxia. La mediana del tiempo de latencia entre la aparición de la rinitis y el asma fue de 8 meses. Ocho pacientes referían una historia clara de alergia a frutas. En las pruebas cutáneas, 10 estaban sensibilizados a papaína, 9 a castaña, 7 a kiwi y 5 a plátano. En la tabla 1 se presentan los datos demográficos y los resultados más relevantes del estudio clí nico-inmunológico. La mayoría de los pacientes fueron estudiados a los pocos meses del inicio de los síntomas bronquiales, si bien el periodo de exposición al látex fue muy variable. No existían diferencias significativas en estos valores de acuerdo a la clínica de los pacientes, si bien los que presentaron manifestaciones de anafilaxia tenían unos niveles de IgE específicos más elevados (clases 3 ó 4), pero esta diferencia no alcanzó significación estadística. La intensidad de la clínica del asma de los pacientes fue leve-moderada, con una mediana de puntuación de 4,3 puntos. La mediana del valor basal del 
Tabla 1. Datos demográficos y clínico-inmunológicos de los pacientes. Los valores se expresan como porcentaje o como la mediana (el rango se indica entre paréntesis).

\begin{tabular}{ll}
\hline $\mathrm{n}^{\mathrm{o}}$ pacientes & 12 \\
\hline Edad & $30(22-54)$ \\
Sexo (\% hombres) & $26 \%$ \\
Atopia & $42 \%$ \\
Años exposición & $6,5(1-30)$ \\
Periodo latencia & $4(0,25-27)$ \\
VEMS basal $\%$ ) & $95 \%(82-126)$ \\
PD20 metacolina & $6 \mu \mathrm{mol}(0,60-12)$ \\
Pruebas cutáneas & \\
(punto final) mg/ml & $0,02(0,01-1,75)$ \\
IgE específica $\mathrm{kU} / \mathrm{L}$ & $5,5(0,77-56,6)$ \\
P. conjuntival $\mathrm{mg} / \mathrm{ml}$ & $0,1(0,01-1)$ \\
\hline
\end{tabular}

VEMS fue del 95\%, presentado la mayoría de los pacientes valores normales u obstrucción leve. La mediana de la $\mathrm{PD}_{20}$ metacolina fue $6 \mathrm{mmol}$ acumulados. Tres pacientes presentaron valores por encima del rango asmático (12 mmol). Los resultados individuales de la provocación bronquial específica se presentan en la tabla 2 . Como se observa, la concentración a la que se registraron respuestas de broncoconstricción iguales o superiores al $20 \%$ fue muy baja. La caída máxima inmediata del VEMS registrada fue del $43 \%$, seguida de otra del $39 \%$ en un paciente con historia de anafilaxia. Todos los pacientes presentaron respuestas asmáticas inmediatas y no se registró ninguna respuesta tardía o dual. Sin embargo, en los dos pacientes en que realizamos una inducción de esputo basal y a las 24 horas tras la provocación el recuento diferencial de células mostró un incremento del porcentaje de eosinófilos del 5 al $39 \%$ y del 3 al $21 \%$, respectivamente. No observamos ninguna complicación, ni síntomas distintos de los respiratorios, incluso en los pacientes que presentaron episodios de anafilaxia. No encontramos correlación entre la provocación bronquial y la sensibilidad cutánea al alergeno, con los niveles de IgE específica frente a látex o con la reactividad bronquial frente a metacolina. Además de cuantificar los cambios del calibre bronquial, la
Tabla 2. Resultados individuales de la provocación bronquial específica.

\begin{tabular}{lcc}
\hline $\mathrm{n}$ & PC20 látex (mg/ml) & Caída máxima VEMS (\%) \\
\hline 1 & 0,87 & 21 \\
2 & 0,87 & 21 \\
3 & 0,20 & 23 \\
4 & 0,81 & 43 \\
5 & 0,05 & 21 \\
6 & 0,15 & $24^{*}$ \\
7 & 0,87 & 21 \\
8 & 0,60 & $39^{*}$ \\
9 & 1,60 & 27 \\
10 & 0,43 & $21^{*}$ \\
11 & 0,30 & 27 \\
12 & 0,01 & 32 \\
\hline
\end{tabular}

${ }^{*}$ Pacientes con historia de anafilaxia

monitorización de parámetros de inflamación de la vía aérea a través de técnicas no invasivas, como la inducción de esputo, puede aportar información importante tanto en el momento del diagnóstico como en el seguimiento de paciente. A pesar de no registrarse respuestas asmáticas tar días, en los dos pacientes en que se realizó la inducción de esputo a las 24 horas se constató un incremento significativo del número de eosinófilos en la vía aérea.

En conclusión, esta modalidad de provocación bronquial con extracto acuoso de látex parece segura, al menos en manos experimentadas. Si bien se aleja notablemente de la forma de exposición natural al alergeno en el ambiente laboral, puede tener utilidad en el diagnóstico y seguimiento de estos pacientes.

\section{En cámara de provocación}

Pisati y $\mathrm{col}^{40}$ prepararon un extracto de guante de látex al $5 \% \mathrm{p} / \mathrm{v}$ y lo aerosolizaron mediante un nebulizador que producía gotas $<20 \mathrm{~mm}$ de diámetro aerodinámico en una cámara de $7 \mathrm{~m}^{3}$, cuando el paciente no se encontraba en la misma. A continuación entraba el paciente y permanecía en la cámara durante 15 minutos o menos si presentaba una respuesta asmática antes de ese tiempo. Se medía el VEMS a los $5,10,15$ $30,60,90$ y 120 minutos y se consideraba 
positiva una caída del VEMS $>15 \%$. Al finalizar se monitorizaba el VEMS cada hora durante las 8 horas siguientes. Si la caída era $<15 \%$ se procedía a nebulizar una solución del extracto 10 veces más concentrada durante 2 minutos. La concentración inicial era una dilución $1 / 10$ de la predeterminada en la titulación cutánea a punto final y la concentración más concentrada el extracto de guante de látex sin diluir.

\section{Provocación con aerosol de polvo de guantes}

\section{Sin cuantificación de aeroalergeno de látex}

La provocación bronquial manipulando o sacudiendo guantes de látex fue descrita inicialmente por Jaeger y $\mathrm{col}^{9}$, haciendo a los pacientes manejar un número creciente de guantes de látex hasta un máximo de 20 pares de guantes durante 60 minutos. En un primer paso los pacientes $(n=18)$ sacudían 20 pares de guantes de material sintético empolvados con almidón de maíz durante 30 minutos en una cámara pequeña. Posteriormente los pacientes manejaban un número creciente de guantes de látex hasta un máximo de 20 pares de guantes durante 60 minutos. Los pacientes llevaban puestos guantes sintéticos durante la prueba para evitar el contacto cutáneo directo. Se midió la resistencia de la vía aérea $\left(\mathrm{sR}_{\mathrm{aw}}\right)$, considerándose positivo un aumento de más del $100 \%$ sobre el basal, y el volumen de gas intratorácico a intervalos periódicos hasta 5 horas después de finalizar la provocación. La inhalación del polvo de los guantes ocasionó síntomas alérgicos con aumento de la resistencia de la vía aérea en cinco de los 18 pacientes.

Posteriormente este método fue estandarizado por Vandenplas y $\mathrm{col}^{12}$, en una prueba en la que los pacientes abrían las bolsas de los guantes y sacudían cada par de guantes durante 3 minutos. En un primer día control los pacientes manejaban guantes de vinilo durante una hora en una cámara de $5 \mathrm{~m}^{3}$ para confirmar que la variación espontánea del VEMS era menor del $10 \%$. Se realizaban espirometrías antes de la provocación, cada 15 minutos duran- te la primera hora, cada media hora la segunda hora y horarias hasta 6 horas después de finalizar la provocación. La PC20 histamina se midió al final del día control. En el segundo día los pacientes manipulaban guantes empolvados de látex. Para ello, los pacientes abrían las bolsas de los guantes y sacudían cada par de guantes durante 3 minutos. La duración de la exposición se aumentaba progresivamente hasta que se producía una caída del VEMS del $20 \%$ o mayor o hasta alcanzar un tiempo acumulado de exposición máximo de 4 horas. Cuando la provocación inhalatoria con látex no producía variaciones significativas del VEMS se determinaba nuevamente la $\mathrm{PC}_{20}$ histamina a las 6 horas, considerándose significativo una variación mayor de 3 veces en la misma. No realizaron determinación de la concentración ambiental de polvo ni alergenos de látex.

Recientemente, Kurtz y col ${ }^{41}$ han propuesto un método de provocación semicuantitativo utilizando una cámara que el paciente se coloca a modo de escafandra (hooded exposure chamber), generándose a continuación un aerosol de almidón de maíz con látex obtenido a partir de guantes empolvados. La concentración inhalada se aumenta progresivamente en pasos sucesivos doblando la dosis, de forma simple ciego. Observaron repuestas positivas en 22 de los 23 pacientes provocados, aunque el criterio de positividad fue muy laxo (disminución del PEF de un 15\%, o de un $10 \%$ con aumento de los síntomas nasooculares o bronquiales).

\section{Con cuantificación de aeroalergeno de látex}

Laoprasert y $\operatorname{col}^{42}$ han utilizado un método de generar aerosol de látex similar al previamente descrito ${ }^{12}$, con pequeñas variaciones, para determinar la eficacia de determinadas intervenciones ambientales, como la utilización de cascos con filtros HEPA. Estos autores realizaban la provocación usando y sacudiendo guantes de látex, un par cada 3 minutos, en una cámara de $20 \mathrm{~m}^{3}$. Para ello los pacientes se ponían los guantes y efectuaban sacudidas bruscas durante un minuto con cada guante alternativamente antes de tirarlos. 
Debajo de los guantes de látex llevaban guantes de vinilo. La provocación duró un máximo de una hora (20 pares de guantes). Se realizaba un día control utilizando únicamente guantes de vinilo. Se registraron los síntomas y signos clínicos basales, incluyendo sibilancias, disnea, tos, obstrucción nasal, estornudos, rinorrea y los síntomas oculares en una escala de puntuación semicuantitativa ( 0 a 3$)$. La puntuación de síntomas también se evaluó cada 15 minutos durante la provocación y al final de la tarde tras cada provocación. Se calculó un valor medio de síntomas para las 7 puntuaciones analizadas, tanto basal como el máximo obtenido durante la provocación. Los cambios en la puntuación de síntomas se calcularon restando el valor medio de las puntuaciones basales de síntomas de la media de las puntuaciones máximas obtenidas durante la provocación. Se midió el VEMS basal y cada 15 minutos durante la provocación. Se trató de determinar el tiempo necesario para obtener una caída del VEMS del $20 \%$. Se realizó determinación de la concentración ambiental de aeroalergeno de látex utilizando bombas de muestreo personales y medidor ambiental de área (Air Sentinel), en el que se cambiaron los filtros cada 15 minutos, obteniéndose una gráfica del tiempo $(0,15,30,45$ y 60 minutos) y la concentración ambiental acumulada de látex en $\mathrm{ng} / \mathrm{m}^{3}$.

En la Fundación Jiménez Díaz se utiliza un método muy similar al descrito anteriormente, pero sin la colocación de ningún tipo de protección (cascos, filtros) que pudiera interferir con la respuesta del paciente ${ }^{43,44}$. Nuestro objetivo era tratar de relacionar las concentraciones del bioaerosol de polvo de guantes de látex que se producen en una cámara de provocación con las mediciones de parámetros biológicos, tal como la función pulmonar, por lo que denominamos a este tipo de exposición provocación inhalatoria cuantificada (PIC).

Realizamos está provocación en 30 pacientes asmáticos (edad media 36 años, rango 21-53), 28 de los cuales eran trabajadores sanitarios, que estaban expuestos de forma más o menos continúa a artículos de látex natural en su lugar de trabajo, en casi todos los casos guantes de látex empolvados. Durante la provocación inhalatoria se monitorizaron los síntomas de rinoconjuntivitis y asma, la función pulmonar, la duración máxima de la exposición y el tipo de reacción asmática (inmediata, tardía o dual). La cámara que nosotros utilizamos tiene $7 \mathrm{~m}^{3}$ y está equipada con un sistema de extracción con filtros HEPA, que permite eliminar por completo las partículas de polvo tras cada prueba de provocación.

Los aerosoles de polvo que contienen las proteínas alergénicas del látex se generan procediendo a darle la vuelta a un par de guantes de látex empolvados, siempre del mismo lote, y sacudiendo cada par durante 3 minutos, tirándolos a continuación. El tiempo de exposición, y por tanto el número de pares de guantes, se aumenta progresivamente durante periodos de 3 , $6,15,30$ y 60 minutos (es decir, 1, 2, 5, 10 y 20 pares de guantes). La concentración de polvo total en la cámara durante cada uno de estos periodos se cuantificó con un medidor láser de aerosoles de polvo (DustTrack, modelo 8520, TSI, EE.UU). Los aeroalergenos del látex se captaban con un medidor ambiental de área volumétrico y filtros de politetrafluoroetileno (Air Sentinel, Quan-Tec-Air, Inc., Rochester, EE.UU). El flujo de captación de este medidor era de 2,5 litros por segundo a través del filtro con un tamaño de poro de 0,3 micras y una eficiencia del 99,9\%. Los aeroalergenos de látex se cuantificaban $\left(\mathrm{ng} / \mathrm{m}^{3}\right)$ como la media ponderada en el tiempo mediante un ensayo de inhibición competitiva de la fijación de IgE utilizando suero de pacientes alérgicos al látex ${ }^{21}$. Los guantes que se utilizaron en las provocaciones se analizaron para medir su contenido total de alergenos de látex. Las mediciones de los parámetros espirométricos se realizaron justo antes de la provocación específica y posteriormente a intervalos regulares durante al menos las 9 horas siguientes y de nuevo a las 24 horas. Previamente a la provocación inhalatoria con látex se realizó un día de exposición control en el que los pacientes manipulaban guantes de vinilo hasta un máximo de 15 minutos. La duración de la exposición a los guantes de látex se aumentaba progresivamente hasta que el 
VEMS caía un $20 \%$ o hasta que se alcanzaba un periodo de exposición de máxima acumulada de 114 minutos.

El síntoma que presentaron más frecuentemente estos 30 pacientes con la PIC fue la rinoconjuntivitis $(90 \%)$ seguida del asma (63\%). Un paciente desarrolló tos debida a bronquitis eosinofílica ${ }^{43}$. Las respuestas asmáticas fueron de tipo inmediato en 15 pacientes, duales en 3 casos y se observó una respuesta tardía aislada en otro paciente. La duración de la exposición necesaria para causar una reacción asmática fue $3,6,15,30$ y 60 minutos en $2,5,7$, 2 y 3 pacientes, respectivamente. Los guantes empolvados no quirúrgicos que se utilizaron (siempre del mismo lote) tenían un gran contenido alergénico de látex (208 $\mathrm{mg}$ por gramo de guante), con los que se conseguían concentraciones estables de aeroalergeno de látex al poco tiempo de comenzar la provocación. Por tanto, la dosis total de látex que se asociaba con la inducción de la repuesta asmática estaba en gran medida condicionada por la duración de la exposición. Las concentraciones de aeroalergeno de látex medidas a diferentes intervalos de tiempo eran reproducibles. Observamos que existía una correlación significativa entre la concentración de polvo total y la concentración de aeroalergeno de látex en la cámara $(\mathrm{r}=0,6$; $\mathrm{p}<0,01)$. Las concentraciones medias de aeroalergeno de látex que se obtuvieron en los pacientes que presentaron una reacción asmática $\left(639 \pm 295 \mathrm{ng} / \mathrm{m}^{3}\right)$ no diferían significativamente de los pacientes que no tuvieron respuestas asmáticas $(611+351$ $\mathrm{ng} / \mathrm{m}^{3}$ ) durante la PIC. Las concentraciones medias de aeroalergeno de látex durante las PIC que indujeron respuestas asmáticas positivas oscilaron entre 199 y 1.107 $\mathrm{ng} / \mathrm{m}^{3}$.

Más del 70\% de los pacientes que tuvieron reacciones asmáticas requirieron un periodo de exposición de 15 minutos o menos. La dosis total acumulada de aeroalergeno de látex inhalada por los pacientes que presentaron respuestas asmáticas osciló entre 25 y 500 ng $(215,7 \pm 168,3)$.

Aunque la respuesta asmática tras exposición al látex es normalmente inmediata, la existencia de respuestas asmáti- cas tardías aisladas tras la provocación con látex ha sido descrita previamente ${ }^{45}$.

El látex además de asma puede provocar otras patologías alérgicas respiratorias como la bronquitis eosinofílica ${ }^{44}$. La bronquitis eosinofílica es una entidad que se presenta como tos no productiva persistente o crónica aislada ${ }^{46}$. Se caracteriza porque no existe obstrucción variable a flujo aéreo, ni variabilidad diaria en los registros de PEF, ni hiperreactividad bronquial a agentes farmacológicos.

\section{Evolución de los pacientes con asma por látex}

Por el momento existen pocos datos sobre la historia natural del asma ocupacional por látex. Los efectos a largo plazo tras disminuir o cesar la exposición al látex en pacientes con asma causada por látex son en buena medida desconocidos Tal como sucede con el asma ocupacional de otra etiología, parece que el látex también puede ocasionar daño respiratorio permanente, incluso tras cesar completamente la exposición al mismo ${ }^{45}$. Brugnami y col ${ }^{45}$ estudiaron a 6 enfermeras alérgicas al látex, 4 de ellas con asma confirmada por prueba de provocación, a las que efectuaron un seguimiento durante un periodo de 7 meses a 7 años. Tres de las pacientes continuaban con síntomas de asma entre 2 y 7 años tras haber cesado la exposición al látex por cambio de puesto de trabajo. No obstante, otros autores han descrito una evolución favorable al cesar la exposición tras un cambio de puesto laboral o al usar guantes sin látex ${ }^{47}$. Recientemente, Vandenplas y $\mathrm{col}^{48}$ han realizado un estudio en el que han comparado los cambios en los parámetros clínicos y en los datos socioeconómicos de los pacientes con asma inducida por látex tras producirse una reducción en la exposición al látex o el cese completo de la misma. En este estudio se incluyeron 36 pacientes diagnosticados de asma causada por látex, confirmado mediante prueba de provocación inhalatoria, en los que se realizó un seguimiento promedio de 56 meses (rango 12 a 92 meses). En todos lo pacientes se realizó una historia clínica y laboral detallada, asî como mediciones de la $\mathrm{PC}_{20}$ histamina al 
principio y al final del estudio. Durante el periodo de seguimiento se obtuvo información sobre la situación laboral, situación económica y calidad de vida. Al final del estudio se encontró que 16 pacientes ya no estaban expuestos al látex, mientras que 20 pacientes habían reducido sustancialmente su exposición laboral a este producto. En los pacientes que evitaron por completo el látex, la gravedad del asma se redujo significativamente $(\mathrm{p}=0,001)$ y la mediana de la $\mathrm{PC}_{20}$ histamina aumentó de $0,4 \mathrm{mg} / \mathrm{mL}$ a $2,3 \mathrm{mg} / \mathrm{mL}(\mathrm{p}=0,002)$. En los pacientes en los que había disminuido su exposición al látex, la gravedad del asma también mejoró de forma significativa (p $<0,001$ ) y la $\mathrm{PC}_{20}$ histamina aumentó de 0,5 $\mathrm{mg} / \mathrm{mL}$ a $2,4 \mathrm{mg} / \mathrm{mL}(\mathrm{p}<0,001)$. El cese total de la exposición al látex se asociada con incapacidad laboral por asma (69\%) y disminución de los ingresos (62\%) más frecuentemente que la reducción de la exposición (35\% y $30 \%$, respectivamente). Estos autores concluyen que la reducción efectiva de la exposición laboral al látex es una alternativa plausible que debería considerarse como una posibilidad razonablemente segura en el tratamiento de estos pacientes, y que llevaría aparejada menos perjuicios socioeconómicos que el cese total de la exposición.

En los pacientes descritos anteriormente estudiados en la Sección de Alergología del Hospital Virgen del Camino ${ }^{49}$, se realizó un seguimiento durante una mediana de 3 años (rango 1-7 años). Un 26\% de los pacientes continuaba en el mismo puesto de trabajo, realizando medidas de evitación, otro $26 \%$ continuaba en labores sanitarias, si bien en distinto puesto de trabajo, y un $48 \%$ habían abandonado completamente la actividad sanitaria. El 16\% de los pacientes se encontraba asintomático sin requerir medicación (todos ellos fuera de la actividad sanitaria), un $32 \%$ requerían broncodilatadores a demanda y un $52 \%$ precisaban corticosteroides inhalados regulares para el control de los síntomas. Tres de estos últimos habían abandonado completamente la actividad sanitaria. Los resultados de la provocación bronquial específica en el momento del diagnóstico no sirvieron para discriminar la evolución de los pacientes. En un paciente se repitió la provocación bronquial específica tras un año de reubicación fuera de la sanidad. En el nuevo estudio solamente se registraron descensos del PEF del 12-18\% entre las 4-10 horas tras la inhalación de la máxima concentración de látex $(2,5$ $\mathrm{mg} / \mathrm{ml}$ ). Sin embargo, en la inducción del esputo tras la provocación específica registramos un incremento importante del número de eosinófilos.

Respecto a los cambios en el grado de sensibilización tras cesar la exposición al látex tampoco existen muchos datos. Allmers y col $^{26}$ estudiaron prospectivamente el efecto de la retirada de los guantes de látex con polvo en un hospital alemán sobre las concentraciones de aeroalergeno de látex y los niveles de IgE específica en los trabajadores alérgicos al látex. La sensibilización al látex se determinó mediante pruebas cutáneas en prick y cuantificación de IgE específica. Las concentraciones ambientales de látex en varias zonas hospitalarias se midieron mediante un ensayo de inhibición competitiva antes y después de la sustitución de los guantes de látex con polvo por guantes sin polvo o guantes de material sintético en diferentes departamentos del hospital. La prevalencia de pruebas cutáneas e IgE específica positivas al látex entre el personal sanitario fue del $8 \%(n=7)$ entre los 90 trabajadores estudiados antes de la intervención ambiental, todos los cuáles tenía síntomas alérgicos con los guantes de látex. Seis de los 7 pacientes sensibilizados presentaron una disminución significativa de los niveles de IgE específica al látex durante el seguimiento un año después. Además, las concentraciones de aeroalergeno de látex, que alcanzaban hasta los $50 \mathrm{ng} / \mathrm{m}^{3}$, a las 24 horas de haber sustituido los guantes de látex con polvo disminuyeron por debajo del límite de detección del ensayo en las áreas con guantes de látex sin polvo o sintéticos ${ }^{26}$. La medicación antiasmática y fármacos antialérgicos pudo retirarse en dos trabajadores con asma ocupacional por látex. Estos resultados demuestran que la eliminación de los guantes de látex con polvo es útil en reducir drásticamente la concentración ambiental de látex y puede permitir a los trabajadores alérgicos que permanezcan en su puesto de trabajo. 
La detección precoz de la sensibilización al látex debería traducirse en medidas de prevención personal, tales como evitar el contacto y la exposición al látex, tanto en el lugar de trabajo como fuera del mismo, lo que también debe incluir la utilización de guantes sin látex, o de látex sin polvo, por los compañeros de trabajo que se encuentren en las mismas áreas. No obstante, estas medidas pueden resultar insuficientes para prevenir la cronificación del asma ocupacional por látex si no se produce la sustitución de los guantes de látex con polvo de forma general en todo el centro de trabajo (hospital, clínica, etc.). La sustitución por guantes de látex sin polvo, guantes sin látex o ambas medidas han demostrado ser eficaces en reducir las concentraciones de látex ambiental a niveles prácticamente indetectables ${ }^{22,28}$.

Recientemente se ha confirmado la efectividad de estas medidas, junto a los programas de educación y vigilancia, en la prevención de la sensibilización al látex en un hospital universitario canadiens $\mathrm{e}^{50} \mathrm{y}$ en el número de casos de asma ocupacional por látex en Ontario ${ }^{51}$. Las ventajas de estas medidas en términos de la relación coste-eficacia han sido confirmadas en otros estudios ${ }^{52}$

\section{BIBLIOGRAFÍA}

1. Carrillo T, Cuevas M, Muñoz T, Hinojosa M, Moneo I. Contact urticaria and rhinitis from latex surgical gloves. Contact Dermatitis 1986; 15: 69-72.

2. Vandenplas O, Charous BL, Tarlo $\mathrm{S}$. Latex allergy. En: Bernstein IL, M C-Y, Malo JL, Bernstein DI, eds. Asthma in the work place. New York: Marcel Dekker, 1999: 425-444.

3. Ross DJ. Ten years of the SWORD project. Surveillance of Work-related and Occupational Respiratory Disease. Clin Exp Allergy 1999; 29: 750-753.

4. Hnizdo E, Esterhuizen TM, ReEs D, LALloo UG Occupational asthma as identified by the surveillance of work-related and occupational respiratory diseases programme in South Africa. Clin Exp Allergy 2001; 31: 32-39.

5. Seaton A, Cherrie B, Turnbull J. Rubber glove asthma. Br Med J (Clin Res Ed) 1988; 296: 531-532.
6. BAUR X, JäGER D. Airborne antigens fron latex gloves [letter]. Lancet 1990; 335: 912.

7. AGIER F, BADIER M, MARTIGNY J, ChARPIN D, VERVLOET D. Latex as aeroallergen. Lancet 1990; 336: 516-517.

8. Marcos C, Lazaro M, Fraj J, Quirce S, De La Hoz B, FERNÁNDEZ-RIVAS M et al. Occupational asthma due to latex surgical gloves. Ann Allergy 1991; 67: 319-323.

9. JAEger D, KLeinhans D, CZuPpon AB, BAur X Latex-specific proteins causing immediatetype cutaneous, nasal, bronchial, and systemic reactions. J Allergy Clin Immunol 1992; 89: 759-768.

10. Bubak ME, ReEd CE, Fransway AF, Yunginger JW, JONES RT, CARLSON CA et al. Allergic reactions to latex among health-care workers. Mayo Clin Proc 1992; 67: 1075-1079.

11. TARLO SM, WONG L, Roos J, Booth N. Occupational asthma caused by latex in a surgical glove manufacturing plant. J Allergy Clin Immunol 1990; 85: 626-631.

12. Vandenplas O, Delwiche JP, Evrard G, Aimont P, VAN DER BREMPT X, JAMART Y et al. Prevalence of occupational asthma due to latex among hospital personnel. Am J Respir Crit Care Med 1995; 151: 54-60.

13. ORFAN NA, ReEd R, DYkEWICZ MS, Ganz M, KOLSKI GB. Occupational asthma in a latex doll manufacturing plant. J Allergy Clin Immunol 1994; 94: 826-830.

14. Pisati G, Baruffini A, Bernabeo F, Falagiani $P$ Environmental and clinical study of latex allergy in a textile factory. J Allergy Clin Immunol 1998; 101: 327-329.

15. Weytjens K, Labrecoue M, Malo JL, Cartier A. Asthma to latex in a seamstress. Allergy 1999; 54: 290-291.

16. Martinez Rivera C, Monso Molas E, Izouierdo RAmirez J, MoRera Prats J. Latex occupational asthma in the production of chewing gum. A case report. Med Clin (Barc) 1998; 111: 399.

17. OwnBY DR. Manifestatios of latex allergy. En: Fink J, ed. Latex allergy. Vol. 15. Philadelphia: Saunders, 1995:3 1-43.

18. Baur X, Ammon J, Chen Z, Beckmann U, Czuppon $\mathrm{AB}$. Health risk in hospitals through airborne allergens for patients presensitised to latex. Lancet 1993; 342: 1148-1149.

19. Sussman GL, Tarlo S, Dolovich J. The spectrum of IgE-mediated responses to latex. JAMA 1991; 265: 2844-2847.

20. Tomazic VJ, Shampaine EL, Lamanna A Withrow TJ, ADKINSON NF JR, HAMILTON RG. Cornstarch powder on latex products is an 
allergen carrier. J Allergy Clin Immunol 1994 93: 751-758

21. SWANSON MC, BubaK ME, Hunt LW, Yunginger JW, WARNER MA, REED CE. Quantification of occupational latex aeroallergens in a medical center. J Allergy Clin Immunol 1994; 94: 445-451.

22. Tarlo SM, Sussman G, Contala A, Swanson MC. Control of airborne latex by use of powderfree latex gloves. J Allergy Clin Immunol 1994; 93: 985-989.

23. Swanson M, Yunginger J, ReEd C. Immunochemical characterization of airborne natural rubber latex allergen in medical and dental office buidings, Healthy Buildings`95 International Conference, Milano, 1995. Vol. 9.

24. HeIlman DK, Jones RT, SWAnson MC, Yunginger JW. A prospective, controlled study showing that rubber gloves are the major contributor to latex aeroallergen levels in the operating room. J Allergy Clin Immunol 1996; 98: 325330.

25. Baur X, Chen Z, Allmers H. Can a threshold limit value for natural rubber latex airborne allergens be defined? J Allergy Clin Immunol 1998; 101: 24-27.

26. Allmers H, Brehler R, Chen Z, Raulf-Heimsoth M, FELS H, BAUR X. Reduction of latex aeroallergens and latex-specific IgE antibodies in sensitized workers after removal of powdered natural rubber latex gloves in a hospital. J Allergy Clin Immunol 1998; 102: 841-846.

27. SWANSON M, Yunginger J, LAM V, TRuscott W. Airborne natural rubber allergen concentrations within a Malaysian latex glove production facility. En: Indoor Air'99, Proceedings of th 8th International Conference on Indoor Quality and ClimateIndoor Air`99, Edinburgh, 1999. Vol. 1.

28. Charous BL, Schuenemann PJ, Swanson MC. Passive dispersion of latex aeroallergen in a healthcare facility. Ann Allergy Asthma Immunol 2000; 85: 285-290.

29. Swanson M, Zakharov S, Luss L, BabaKhin A, DuBusKE L. Latex aeroallergen quantification in hospitals of Moscow, Russia. Ann Allergy Asthma Immunol 2001; 87: 307-310.

30. WiLliams PB, BuHR MP, WeBER RW, Volz MA, KOEPKE JW, SELNER JC. Latex allergen in respirable particulate air pollution. J Allergy Clin Immunol 1995; 95: 88-95.

31. Miguel AG, Cass GR, Weiss J, Glovsky MM Latex allergens in tire dust and airborne particles. Environ Health Perspect 1996; 104 1180-1186.
32. VANDENPlas O, BinaRd-Van CANGH F, BRUMagne A, Caroyer JM, Thimpont J, SoHy $\mathrm{C}$ et al. Occupational asthma in symptomatic workers exposed to natural rubber latex: evaluation of diagnostic procedures. J Allergy Clin Immunol 2001; 107: 542-547.

33. Baur X, Huber H, Degens PO, Allmers H, AMMON J. Relation between occupational asthma case history, bronchial methacholine challenge, and specific challenge test in patients with suspected occupational asthma. Am J Ind Med 1998; 33 : 114-122.

34. Lemiere C, Cartier A, Dolovich J, Chan-Yeung M, Grammer L, Ghezzo $\mathrm{H}$ et al. Outcome of specific bronchial responsiveness to occupational agents after removal from exposure. Am J Respir Crit Care Med 1996; 154: 329-333.

35. Kari O, lauerma $\mathrm{H}$, Alenius $\mathrm{H}$, Pauloso $\mathrm{T}$ REUNALA T. Ocular challenge with natural rubber latex. J Allergy Clin Immunol 1997; 99 . 343

36. PALCZynSKi C, WALUSIAK J, RUTA U, GORSKi P. Nasal provocation test in the diagnosis of natural rubber latex allergy. Allergy 2000; 55 $34-41$

37. PALCZynski C, WALUSIAK J, RUTA U, GoRSKI P Occupational allergy to latex-life threatening reactions in health care workers. Report of three cases. Int J Occup Med Environ Health 1997; 10: 297-301.

38. Ho A, Chan H, Tse KS, Chan-Yeung M Occupational asthma due to latex in health care workers. Thorax 1996; 51: 1280-1282.

39. Acero S, Tabar A, Alvarez M, Garcia B, ECHECHIPIA, OlAGUIBEL J. Occupational asthma due to natural rubber latex. specific inhalation challenge test and evolution. J Invest Allergol Clin Immunol 2003; 13:(in press).

40. Pisati G, Baruffini A, Bernabeo F, Stanizzi R BRONCHIAL provocation testing in the diagnosis of occupational asthma due to latex surgical gloves. Eur Respir J 1994; 7: 332-336.

41. KuRTZ KM, HAMiLTON RG, SCHAEFER JA, AdKINSON NF, JR. A hooded exposure chamber method for semiquantitative latex aeroallergen challenge. J Allergy Clin Immunol 2001; 107 178-184.

42. LAOPRASERT N, SWANSON MC, JONES RT, SCHROEDER DR YUNGINGER JW. Inhalation challenge testing of latex-sensitive health care workers and the effectiveness of laminar flow HEPA-filtered helmets in reducing rhinoconjunctival and asthmatic 
reactions. J Allergy Clin Immunol 1998; 102: 998-1004.

43. Quirce S, Swanson M, Fernández-Nieto M, DE las Heras M, Cuesta J, Sastre J. Quantified environmental challenge (QEC) using rubber glove cornstarch aerosols compromises pulmonary function in patients with natura rubber latex allergy. J Allergy Clin Immunol 2002; 109: 110.

44. Quirce S, Fernandez-Nieto M, DE Miguel J, SASTRE J. Chronic cough due to latex-induced eosinophilic bronchitis. J Allergy Clin Immunol 2001; 108: 143.

45. Brugnami G, Marabini A, Siracusa A, Abbritti G. Work-related late asthmatic response induced by latex allergy. J Allergy Clin Immunol 1995; 96: 457-464.

46. Gibson PG, Dolovich J, Denburg J, Ramsdale EH, HARGREAVE FE. Chronic cough: eosinophilic bronchitis without asthma. Lancet 1989; 1: 1346-1348.

47. Valentino M, Pizzichini MA, Monaco F, Governa M. Latex-induced asthma in four healthcare workers in a regional hospital. Occup Med (Lond) 1994; 44: 161-164.

48. Vandenplas O, Jamart J, DelWiche JP, Evrard G, LARBANOIS A. Occupational asthma caused by natural rubber latex: outcome according to cessation or reduction of exposure. J Allergy Clin Immunol 2002; 109: 125-130.

49. OlaguiBel JM. Asma ocupacional. Alergenos de alto peso molecular: látex. Alergo Aragon. Formigal 1997.

50. Tarlo SM, Easty A, Eubanks K, Parsons CR, Min F, JUVET $S$ et al. Outcomes of a natural rubber latex control program in an Ontario teaching hospital. J Allergy Clin Immunol 2001; 108 : 628-633.

51. Liss GM, TARLO SM. Natural rubber latexrelated occupational asthma: association with interventions and glove changes over time. Am J Ind Med 2001; 40: 347-353.

52. Phillips VL, Goodrich MA, SULLIVAN TJ. Health care worker disability due to latex allergy and asthma: a cost analysis. Am J Public Health 1999; 89: 1024-1028. 